\title{
Decoding of periodontal screening and recording index
}

\author{
Dler Ali Kursheed $^{(1)}$; Faraedon Mohidden Zardaw ${ }^{(1)}$; Hadi Mohammad Ismail ${ }^{(1)}$; \\ Aras Maruf Rauf ${ }^{(1)}$; Sarhang Sarwat Gul ${ }^{(1)}$
}

\begin{abstract}
Background and Objective: This study based on decoding of the original periodontal screening and recording (PSR) index by adding some additional clinical variables and preserving its simplicity. This study aimed to decode PSR index to reveal all the percentage of variables masked by each code in PSR index.

Methods: In this interventional clinical study, PSR criteria were obtained from 50 participants plus decoding of each PSR code such as percentage of bleeding (BI), calculus (Cal) and pocket depth (PD) in each sextant. Further modification of PSR was involved adding few extra variables such as stain (St), over-hanged restorations (Ov) and crowding $(\mathrm{Cr})$ to each sextant. The average of PSR codes and following decoding in each subject was calculated and compared to show the percentage of masking. The study was conducted in B\&R Dental Center (March-October 2019).

Results: PSR index and decoding of the variables were collected from 50 subjects. PSR code 2 was the most prevalent code (62.61\%) followed by code $1(16.83 \%)$ and code $3(10.1 \%)$. Furthermore, BI, St, Cal and Ov were the most frequent variables recorded among all sextants. Decoding of code 2 , showed that $76.8 \%$ of BI was masked by PSR index. Whereas, PSR code 3 masked $96.2 \%$ of variables of PSR code 1 and 2 (BI, stain, calculus, over-hanged restoration and crowding). Finally, 98.6\% of variables in PSR code 1, 2 and 3 were masked by PSR code 4.

Conclusions: Decoding the PSR index by exposing the masked variables and adding few new variables will develop more informative and inclusive system of examination and diagnosis along with keeping the simplicity of the other indices of periodontal screening such as PSR index.
\end{abstract}

Keywords: Decoding, PSR index, Periodontal examination, Screening.

\footnotetext{
${ }^{(1)}$ College of Dentistry, University of Sulaimani, Sulaimani, Iraq

Corresponding author: Dler Ali Kursheed. Mobile: 009647701424616. Email: dler.khursheed@univsul.edu.iq
}

\section{Introduction}

Diagnosis of periodontal disease usually requires comprehensive periodontal examination including full mouth periodontal tissue probing and measurement of attachment levels. ${ }^{1}$ It is usually timeconsuming procedure and requires high operator skill. ${ }^{2-4}$ In a busy dental practice, it will, therefore, be difficult to carry out full periodontal examination for every dentate patient. ${ }^{5}$ The Community Periodontal Index of Treatment Needs (CPITN) was proposed by Ainamo et al 1982 and determined by the World Health Organization (WHO) as a tool for assessment of periodontal diseases and treatment needs in epidemiologic survey. ${ }^{6} \mathrm{~A}$ shortcoming of this screening instrument is that no attempt was made to score the degree of attachment loss, mobility and furcation involvement. ${ }^{7-9}$ The two most recognized examination tools are undoubtedly the Basic Periodontal Examination (BPE) announced by the British Society of Periodontology and the Periodontal Screening and Recording (PSR) proposed jointly by the American Dental Association and American Academy of Periodontology. ${ }^{7,10}$ Both organizations recommend applying these systems for every patient annually if they are demanding a comprehensive periodontal examination. ${ }^{1}$ Since they developed no suggestion or 
modification has been proposed for any of these indices.

Similar to CPITN, periodontal screening and recording employing a special probe that is WHO probe designed as a ballshaped tip of $0.5 \mathrm{~mm}$ diameter with a black band of $2 \mathrm{~mm}$. The distance from the tip of the ball to the beginning black band measures 3.5 and to the end of the black band $5.5 \mathrm{~mm}$. The probe is "walked around" the teeth in the gingival sulcus for each sextant using a light pressure of 20-25 grams. The highest PSR score per sextant is recorded. The PSR codes with only shallow probing depths less than $3.5 \mathrm{~mm}$ at all tooth sites are assigned as either 0,1 or 2 , according to the presence or absence of bleeding on probing, dental calculus deposits, and/or defective dental restoration margins in the sextant respectively. ${ }^{12,13}$ Sextants with deeper probing depths ranging between 3.5 to $5.5 \mathrm{~mm}$ whereby the black band is partially veiled by the pocket wall is assigned as PSR code 3, whereas sextants with at least one probing depth greater than $5.5 \mathrm{~mm}$, where the black band is totally obscured, receives a PSR index score 4 . In addition to the 0 to 4 grading scale, a Code * (asterisk) is added to PSR scores when additional periodontal parameters are detected in any of the sextant such as furcation involvements, excessive tooth mobility and gingival recession. ${ }^{11,13}$ PSR index considers and records the maximum score in the sextant, however, the examiner measures all data in the sextant, but the most sever record is constantly designated and recorded.

The role of bacterial plaque in initiating gingival inflammation is undisputable., 74 However, because most patients on the day and before visiting their dentists, they brush their teeth well, it may be difficult to see dental plaque on the teeth. But bleeding is a good evidence for the presence of long exposure of gingival tissues to plaque accumulation. Furthermore, appearance of the dentition is of concern to a large number of people seeking dental treatment and the color of the teeth is of particular cosmetic importance. ${ }^{15,16}$ Although stain does not cause gingival inflammation as dental plaque does, but it should be removed for esthetic purposes and its removal from the surface of the teeth surfaces adding extra time and cost to the procedure. Therefore, and for professional considerations it is necessary to be added to the other variables as a unique variable.

Periodontal Screening and Recording ordinarily exhibits extent and severity of periodontal diseases rather than defining other important variables and features required for appropriate diagnosis and treatment strategy. ${ }^{7}$ Furthermore, the index does not give an idea about time and cost of each treatment approach, which is necessary to be determined before starting initial treatment.

The validity of hierarchical method of measuring CPITN has been questioned and Benigeri et al. 2000 reported that about $30 \%$ of the teeth with calculus did not have bleeding and one fourth of teeth with deep periodontal pockets did not have calculus. PSR index was announced in 1992 designed to initiate the promotion, prevention, and early treatment of periodontal diseases by introducing a simplified screening method that met authorized dental recording requirements. Only three years later exactly in 1995 Khocht et al, found that PSR underestimates and/or overestimates the actual sextants extent when compared with full periodontal examination and charting. ${ }^{9}$ In light of the discrepancy between PSR and full mouth measurements, some wariness about the exact extent and severity of periodontal condition will develop and consequently time needed for treatment of each individual condition will be disturbed. Therefore, the aim of this study was based on decoding of the original PSR index in an attempt to reveal all the existed data that are masked by the simplicity of PSR index. Furthermore, decoding process involved adding some supplementary clinical variables, preserving PSR simplicity and giving a clear image about the periodontal status.

\section{Methods}

Fifty subjects (16 males and 32 females) were recruited in this study with nonhierarchical manner for the purpose of decoding PSR index. The patients were referred for the purpose of professional dental cleaning (scaling and polishing) and they did not have history of professional 
therapy the last 6 months. Ethical approval obtained from ethical committee of Faculty of medicine, Dentistry and Pharmacy, University of Sulaimani and the study conducted between March and October 2019 in B\&R Dental Center, Sulaymaniyah city, Iraq. Patient information sheet were provided for potential participant and then consent from were obtained. PSR index was recorded for each sextant using WHO probe as follow: code 0 when there is pocket depth (PD) $<3.5 \mathrm{~mm}$, no bleeding and no calculus, code 1 includes $P P D<3.5 \mathrm{~mm}$, no calculus but there is bleeding on probing, code 2 includes $\mathrm{PD}<3.5 \mathrm{~mm}$, presence of bleeding and calculus, code 3 includes PD of $3.5-5.5 \mathrm{~mm}$ and code 4 includes PD $>5.5 \mathrm{~mm}$. Furthermore, the PSR chart also involved asterisk symbols for tooth mobility $(\mathrm{M})$, furcation involvement $(\mathrm{F})$ and gingival recession (R). Finally, the highest PSR code was recorded for each sextant. Decoding of PSR index (Figure 1) were performed that all variables were recorded per sextant to avoid masking of other variables by one variable. For example, in sextant with PSR code 3, all other variables if present (BI, St. $\mathrm{Cal}, \mathrm{Ov}$ and $\mathrm{Cr}$ ) were recorded beside PD of $3.5-5.5 \mathrm{~mm}$, furcation, recession and mobility. All variables were recorded in each sextant separately and their frequency within each sextant were calculated.

Statistical analysis. Frequency of original PSR index were calculated for all sextants and then after decoding, frequency of all variables (BI, St, Cal, Ov, Cr, PD, also R, F and $\mathrm{M}$ ) were calculated for all sextant as well. The percentage of masking were identified by dividing frequency each PSR code to the corresponding total number of examined variables. all calculations were conducted using the SPSS software package (version 21; SPSS Inc., Chicago, IL, USA). The null hypothesis was that PSR index does not mask any examined variables.

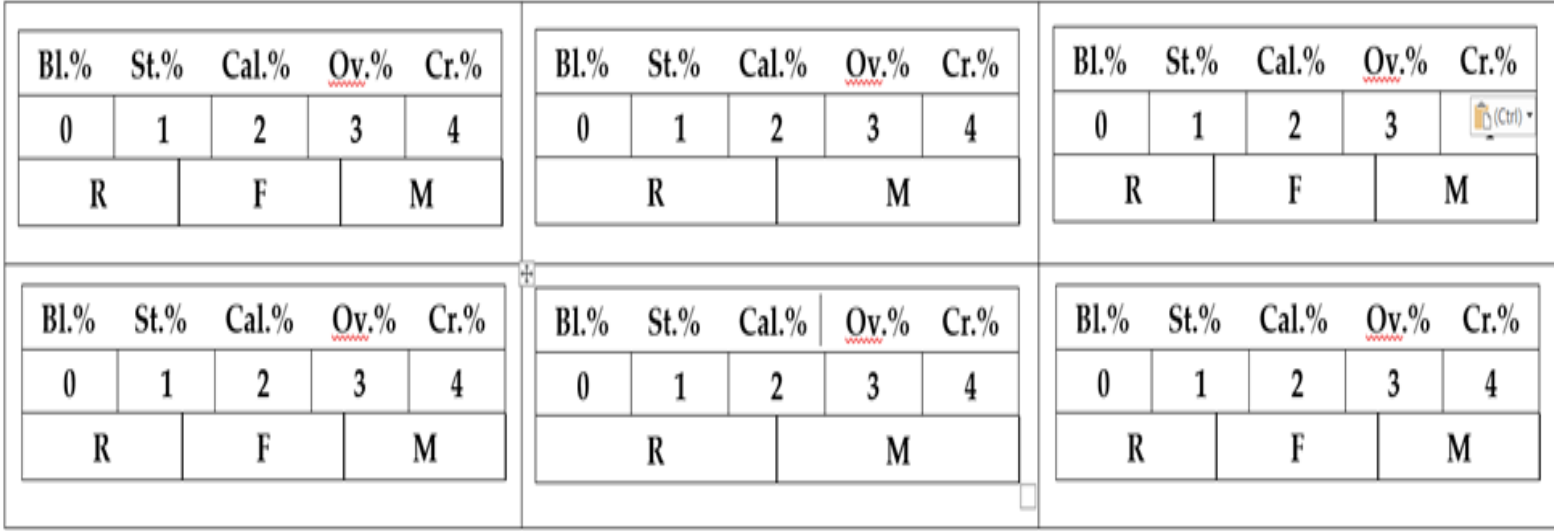

Figure 1: The expanded Periodontal record system by adding further variables and symbols to the original system. BI, Bleeding; St, stain; Cal, Calculus, Ov, Overhanging restoration, $\mathrm{Cr}$, crowding. $\mathrm{R}$, Gingival recession; F, Furcation involvements; $M$, Tooth mobility.

\section{Results}

Fifty subjects (16 males and 32 females) and PSR index were collected. PSR code 2 was the highest amongst them (62.61\%) followed by code $1(16.3 \%)$ and code $3(10.1 \%)$ (Table 1 and Figure 2). Furthermore, the decoded variables (BI, St, Cal, Cr, Ov, PD 3.5$5.5 \mathrm{~mm}$ and $\mathrm{PD}>5.5 \mathrm{~mm}$ ) were also recorded in all sextants for all subjects. Stain was the highest variable with $88 \%$ and BI was $85.33 \%$ followed by $\mathrm{Cal}(56.33 \%)$ and $\mathrm{Ov}$ (23.66\%). While, PD between 3.5-5.5mm was $10.05 \%$ and $\mathrm{PD}>5.5 \mathrm{~mm}$ was $4.05 \%$, the least frequent variable (Table 1).
UR, Upper right; UM, Upper middle sextant; UL, Upper left; LR, Lower right; LM, Lower middle; LL, Lower left

The sum of PSR code 2 in all sextants was 375.5 and the sum of the variables that can be masked by PSR code was 1612 . The total revealed data by PSR code 2 was $23.2 \%$ $(1612 / 375.5)$, thus the proportion of masking by PSR code 2 was $76.8 \%$ (Table 2). On the other hand, percentage of masking by PSR code 3 and code 4 were $96.2 \%$ (Table 3 ) and $98.6 \%$ (Table 4), respectively. Finally, as the code of PSR increase, the percentage of masking increase as well. 
Table 1: Proportion of each PSR code and distribution of the supplementary variables in all sextants.

\begin{tabular}{|c|c|c|c|c|c|c|c|c|}
\hline & & \multicolumn{6}{|c|}{ Sextant (\%) } & \multirow{2}{*}{$\begin{array}{l}\text { Total } \\
\text { (\%) }\end{array}$} \\
\hline & & UR & UM & UL & LR & LM & LL & \\
\hline \multirow{5}{*}{ PSR Code } & 0 & 10 & 12 & 4.1 & 6.1 & 2 & 4.1 & 6.38 \\
\hline & 1 & 24 & 22 & 16.3 & 20.4 & 2 & 16.3 & 16.83 \\
\hline & 2 & 52 & 52 & 71.4 & 55.1 & 86 & 59.2 & 62.61 \\
\hline & 3 & 12 & 10 & 2 & 14.3 & 6 & 16.3 & 10.1 \\
\hline & 4 & 2 & 4 & 6.1 & 4.1 & 4 & 4.1 & 4.05 \\
\hline \multirow{7}{*}{$\begin{array}{l}\text { Supplemen- } \\
\text { tary variables } \\
\text { and PSR }\end{array}$} & Bleeding & 84 & 88 & 88 & 84 & 76 & 92 & 85.33 \\
\hline & Stain & 82 & 88 & 90 & 82 & 98 & 88 & 88 \\
\hline & Calculus & 42 & 40 & 54 & 60 & 76 & 66 & 56.33 \\
\hline & Crowding & 4 & 28 & 6 & 12 & 28 & 14 & 15.33 \\
\hline & Overhang & 26 & 36 & 26 & 24 & 12 & 18 & 23.66 \\
\hline & Score 3 only & 12 & 10 & 4 & 14.3 & 6 & 14 & 10.05 \\
\hline & Score 4 only & 2 & 4 & 6.1 & 4.1 & 4 & 4.1 & 4.05 \\
\hline
\end{tabular}

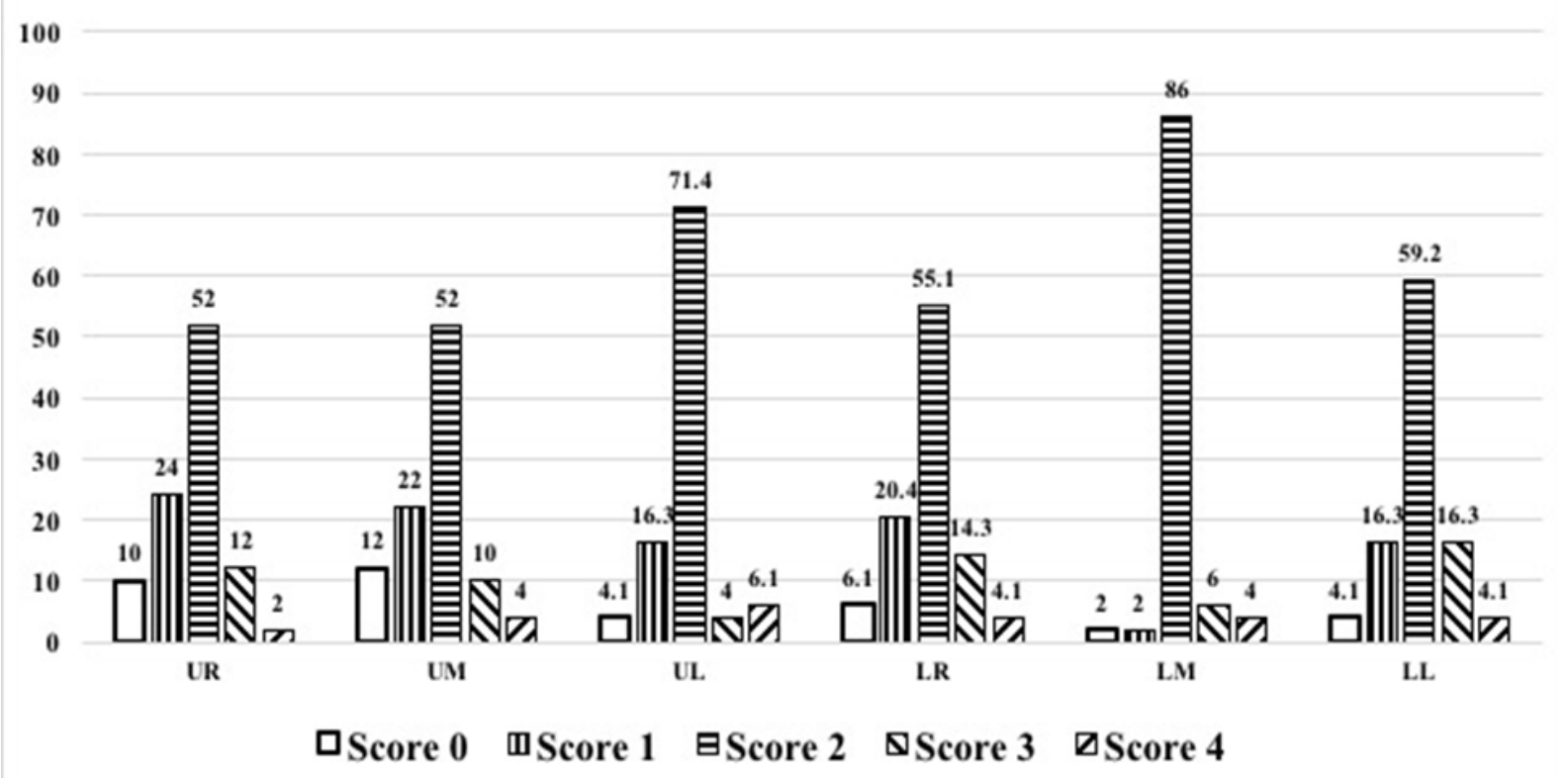

Figure 2: Percentage of each PSR code in all sextants of the study sample.

Table 2: Percentage of masking by PSR code 2. UR, Upper right; UM, Upper middle sextant; UL, Upper left; LR, Lower right; LM, Lower middle; LL, Lower left

\begin{tabular}{|c|c|c|c|c|c|c|c|c|}
\hline & \multicolumn{6}{|c|}{ Sextant (\%) } & \multirow{2}{*}{$\begin{array}{c}\text { Appeared } \\
\text { data }\end{array}$} & \multirow{2}{*}{$\begin{array}{c}\text { Masked data } \\
\text { (\%) }\end{array}$} \\
\hline & UR & UM & UL & LR & LM & LL & & \\
\hline PSR code 3 & 12 & 10 & 2 & 14.3 & 6 & 16.3 & 62.6 & \multirow{6}{*}{96.2} \\
\hline Bleeding & 84 & 88 & 88 & 84 & 76 & 92 & \multirow{5}{*}{1612} & \\
\hline Stain & 82 & 88 & 90 & 82 & 98 & 88 & & \\
\hline Calculus & 42 & 40 & 54 & 60 & 76 & 66 & & \\
\hline Crowding & 4 & 28 & 6 & 12 & 28 & 14 & & \\
\hline Overhang & 26 & 36 & 26 & 24 & 12 & 18 & & \\
\hline
\end{tabular}


Table 4: Percentages of masking by PSR code 4. UR, Upper right; UM, Upper middle sextant; UL, Upper left; LR, Lower right; LM, Lower middle; LL, Lower left

\begin{tabular}{|c|c|c|c|c|c|c|c|c|}
\hline & \multicolumn{6}{|c|}{ Sextant (\%) } & \multirow{2}{*}{$\begin{array}{c}\text { Appeared } \\
\text { data }\end{array}$} & \multirow{2}{*}{$\begin{array}{l}\text { Mask } \\
\text { ed } \\
\text { data } \\
\text { (\%) }\end{array}$} \\
\hline & UR & UM & UL & LR & LM & LL & & \\
\hline PSR code 4 & 2 & 4 & 6.1 & 4.1 & 4 & 4.1 & 24.3 & \multirow{7}{*}{98.6} \\
\hline Bleeding & 84 & 88 & 88 & 84 & 76 & 92 & \multirow{6}{*}{1674.6} & \\
\hline Stain & 82 & 88 & 90 & 82 & 98 & 88 & & \\
\hline Calculus & 42 & 40 & 54 & 60 & 76 & 66 & & \\
\hline Crowding & 4 & 28 & 6 & 12 & 28 & 14 & & \\
\hline Overhang & 26 & 36 & 26 & 24 & 12 & 18 & & \\
\hline RSR code 3 & 12 & 10 & 4 & 14.3 & 6 & 16.3 & & \\
\hline
\end{tabular}

\section{Discussion}

Snice 1992, PSR introduced, no any change has been proposed to modify this examination tool. PSR was designed to record periodontal diseases and condition with an easy probing technique and minimum documentation.

Bleeding is very important variable in clinical setting as it may have few impacts at the time of examination that impedes proper recording measurements and profuse bleeding avoids proper scaling and polishing, which takes more time and causes more trauma to the inflamed gingiva. In this case its preferable to start the first session with motivation and oral hygiene instruction then performing the initial periodontal treatment in the next session. ${ }^{17}$

Decoding of this hierarchical method of scoring, brings better understanding of the patient's condition in order to discuss the overall periodontal health condition with the patient and identify further treatment options. This makes PSR index more inclusive to be used as an effective motivational tool in order to achieve patient's compliance. ${ }^{18}$ On the other hand, decoding process will minimize the underestimation of the clinical parameters. As shown in the result section, high percentages of these important clinical variables were masked by the original index enables proper diagnosis, treatment planning and determination of prognosis. The study also showed the drawback of this hierarchical method of screening, for instance score 4 only display pockets more than $5.5 \mathrm{~mm}$ masking all the other variables within the other scores 3, 2 and score1. Perhaps this may lead to overestimation of the periodontal status as it is masking all other parameters and factors related to diagnosis and treatment. Although both calculus and overhanging restorations are plaque retentive factors and recorded in PSR as score 2, but score 2, is not identifying them as different clinical parameters. However, these have different etiologies and they require different treatment strategies. Calculus is formed on the tooth surfaces as a result of improper oral hygiene performance by the patient, whereas, over hanging is an iatrogenic condition produced by the dentist. Therefore, they should be identified separately and each entity should be provided with a different numeral code in the sextant. The current study revealed high percentages of each calculus and overhanging restoration among this small group of study population. Since PSR index developed from the CPITN index which was designed to determine proper treatment plan not to group etiological factors, this modification of PSR index seems to be more 
applicable and closer to the objective of original PSR index. Decoding of score 2, showed high percentage of bleeding, which is an important clinical risk marker for disease activity and progression. ${ }^{19}$ However dental calculus and stain, overhanging restorations and crowding are required different treatment procedures such as prophylactic, restorative and orthodontic treatments. It is acknowledged that flossing the interdental areas will give rather accurate results than inspecting the over-hanged margins with an explorer specially for tooth colored restorations. Therefore, it is recommended to use dental floss instead of using explorer for inspecting overhangs at proximal restorations. The objective of this study was not to develop or suggest a new recording system. However, it reveals few drawbacks in the original PSR index. Decoding process resulted in a modified version of PSR that figuring out further details of clinical periodontal parameters. As mentioned previously in this section, PSR index is masking high percentages of data related to important clinical variables, perhaps this may have a negative influence on the diagnosis and treatment plan required for each individual case. This modification resulted in minimizing the over- and underestimation of the acquired data.

\section{Conclusion}

Within the limitations of this study, it can be concluded that PSR index mask more than $75 \%$ of valuable data and the proportion of masking increase with increasing PSR code. Clinician should be aware when interpreting PSR codes and add some new variables for PSR index can provide further detail to determine accurate diagnosis and treatment planning. Longitudinal studies with larger sample size are encouraged to validate the importance of PSR decoding.

Conflict of Interest: The authors declare no conflict of interest.

\section{References:}

1.Mitchell T. Periodontal Screening and Recording: Early Detection of Periodontal Diseases, dental- care. com. Continuing Education Course, Revised 2014;9.

2. Primal KS, Esther SR, Boehm TK. Periodontal screening and recording (PSR) index scores predict periodontal diagnosis. J Dent App 2014;1(1):8-12

3. Khocht A, Zohn H, Deasy M, Chang KM. Screening for periodontal disease: radiographs vs. PSR. J Am Dent Assoc 1996;127(6):749-56. doi: 10.14219/ jada.archive.1996.0310

\section{Beltrán-Aguilar ED, Eke PI, Thornton-Evans G, Petersen PE. Recording and surveillance sys- tems for periodontal diseases. Periodontol 2000 2012;60(1):40-53. doi:10.1111/j.1600- 0757.2012.00446.x}

5. Internationale FD. A simplified periodontal examination for dental practices. Aust Dent J 1985;30:368-70. doi:10.1111/j.18347819.1985.tb02534.x

6. Ainamo J. Development of the World Health Organization (WHO) community periodontal index of treatment needs (CPITN). Int dent J 1982;32:28191.

7. Preshaw PM. Detection and diagnosis of periodontal conditions amenable to prevention. BMC oral health 2015;15:S5. BioMed Central. doi:10.1186/1472-6831-15-S1-S5

8. Benigeri $M$, Brodeur JM, Payette $M$, Charbonneau A, Ismaïl Al. Community periodontal index of treatment needs and prevalence of periodontal conditions. J Clin Periodontol 2000;27(5):308-12. doi:10.1034/j.1600-051x.2000.027005308.x

9. Khocht A, Zohn H, Deasy M, Chang KM. Assessment of periodontal status with PSR and traditional clinical periodontal examination. J Am Dent Assoc 1995;126(12):1658-65. doi:10.14219/ jada.archive.1995.0115

10. Meers E, Dekeyser C, Favril C, Teughels W, Quirynen $M$, Laleman I. Periodontal screening and referral behaviour of general dental practitioners in Flanders. Clin Oral Invest 2018;22(3):1167-73. doi:10.1007/s00784-017-2212-1

11. Rams TE, Loesche WJ. Relationship between periodontal screening and recording index scores and need for periodontal access surgery. J periodontol 2017;88(10):1042-50. doi:10.1902/ jop. 2017.170070

12. Periodontology American Dental Association and American Academy of Periodontology / American Dental Association. Periodontal Screening and Recording - An Early Detection System [Report] Chicago 2001;1-8.

13. Landry RG, Jean M. Periodontal Screening and Recording (PSR) Index: precursors, utility and limitations in a clinical setting. Int Dent J 2002;52 (1):35-40. 
14. Löe $H$, Theilade $E$, Jensen SB. Experimental gingivitis in man. j periodontol 1965;36(3):177-87. doi:10.1111/j.1875-595x.2002.tb00595.x

15. Watts A, Addy M. Tooth discolouration and staining: tooth discolouration and staining: a review of the literature. British dental journal. 2001;190 (6):309-16. doi:10.1038/sj.bdj.4800959

16. Joiner A. Review of the extrinsic stain removal and enamel/dentine abrasion by a calcium carbonate and perlite containing whitening toothpaste. Int dent j 2006;56(4):175-80. doi:10.1111/ j.1875-595x.2006.tb00091.x
17. Newbrun E. Indices to measure gingival bleeding. J periodontol 1996;67(6):555-61. DOI:10.1902/ jop.1996.67.6.555

18. Charles $\mathrm{CJ}$, Charles $\mathrm{AH}$. Periodontal screening and recording. J Calif Dent Assoc 1994;22(2):43-6.

19. Chapple IL, Mealey BL, Van Dyke TE, Bartold PM, Dommisch $\mathrm{H}$, Eickholz $\mathrm{P}$ et al. Periodontal health and gingival diseases and conditions on an intact and a reduced periodontium: Consensus report of workgroup 1 of the 2017 World Workshop on the Classification of Periodontal and Peri-Implant Diseases and Conditions. J periodontol 2018;89:S7484. doi:10.1111/jcpe.12940 